\title{
Synthesis and characterization of new macromolecule systems for colon-specific drug delivery
}

\author{
M. Galehassadi, M. Mahkam, F. Hosseinzadeh
}

Department of Chemistry, Azarrbaijan University of Tarbiat Moallem Tabriz, IRAN. Email: mmahkam@yahoo.com,mahkam@azaruniv.edu)

(Received: 25 September, 2006; published: 2 March, 2007)

\begin{abstract}
Network polymers containing silyl groups were synthesized. Silyl derivatives of 2-hydroxyethylmethacrylate (HEMA), and methacrylic acid (MAA) were copolymerized with 1,2-bis(vinylphenyl)ethane (BVPE) and divinylbenzene (DVB) as cross-linking agents by radical polymerization using $\alpha, \alpha^{\prime}$-azobis (isobutyronitrile) (AIBN) as initiator to produce network polymers. Then mesalasine (MZ) were loaded to these network polymers, and amount of drug entrapped was determined. In vitro release studies showed that drug delivery property was modified and showed considerable difference in swelling at $\mathrm{pH} 1$ and 7.4. Incorporation of silyl groups in new macromolecule system modified network polymers for drug delivery. Monomers and polymers were characterized by spectroscopic methods.

Keywords: Silyl groups; network polymers; mesalasine; drug delivery; $\mathrm{PH}$ sensitive.
\end{abstract}

\section{Introduction}

Cross-linking is the formation of chemical links between molecular chains to form a three-dimensional network of connected molecules. The vulcanization of rubber using elemental sulfur is an example of cross-linking, converting raw rubber from a weak plastic to a highly resilient elastomer. The strategy of covalent cross-linking is used in several technologies of commercial and scientific interest to control and enhance the properties of the resulting polymer system or interface, such as thermosets and coatings [1-3]. Cross-linking has been employed in the synthesis of ion-exchange resins and stimuli-responsive hydrogels made from polymer molecules containing polar groups. As polyelectrolytes, hydrogels are inherently water soluble. To make them insoluble, they are chemically cross-linked during manufacture or by a second reaction following that of polymerization of the starting monomers. The degree of cross-linking, quantified in terms of the crosslink density, together with the details of the molecular structure, have a profound impact on the swelling characteristics of the cross-linked system. These reagents were used in biodegradable micelle compositions for carrying and releasing drugs and other active agents for therapeutic or other medical uses. For example for preparing biocleavable or biodegradable micelle compositions that are coupled to biorecognition molecules for targeting the delivery of drugs to their site of action [4].

In general, polymer therapeutics refers to the use of a polymer as a component of a pharmaceutical formulation to improve the efficacy of a drug or gene. Knowledge of the properties of polymers, and polymeric systems, is of fundamental importance in the pharmaceutical sciences and, in particular, their application in polymeric therapeutics. This new, multi-disciplined field has evolved through the development 
of non-viral gene delivery systems, "smart" polymeric gels, polymer conjugates, liposomes, and other micro and nanoparticluate systems such as, block copolymer micelles and self-assembling complexes.

To design effective polymer therapeutics it is necessary to combine leading edge polymer chemistry with pharmaceutics as well as understanding of behavior of macromolecules at the level of the body and cell. This area of research and technology development has attracted tremendous attention during the last two decades and it is expected that it will continue to grow in importance.

Controlled-release drug delivery systems have the potential to provide continuous drug release (i.e., zero-order kinetics), in which blood levels of drugs would remain constant throughout the delivery period [5]. By contrast, injected drugs are cleared by first-order kinetics, so that initial high levels of the drug after initial administration decreases exponentially over time. The potential therapeutic advantages of continuous-release drug delivery systems are significant and encompass in vivo predictability of release rates; minimized peak plasma levels and thereby reduced risk of adverse reactions; predictable and extended duration of action; and reduced inconvenience of frequent dosing and thereby improved patient compliance [6].

Drug delivery and pharmacokinetics have been important elements of biomedical engineering practice for many decades. This long-standing interest is now coupled with rapid progress in both the engineering and biological aspects of drug delivery, making this an appropriate study. In this paper, we prepared new polymer systems and studied their drug delivery using 5-aminosalicylic acid (5-ASA) (mesalasine) as a model drug.

To achieve successful colonic delivery, a drug needs to be protected from absorption of the environment of the upper gastrointestinal tract (GIT) and then be abruptly released into the proximal colon, which is considered the optimum site for colontargeted delivery of drugs. These requirements have prompted the development of polymeric systems that swell minimally under acidic conditions but extensively in basic intestinal medium.

The composition of the polymer determines whether it's a neutral or ionic network and furthermore, its hydrophilic/hydrophobic characteristics. Ionic hydrogels, which could be cationic, containing basic functional groups or anionic, containing acidic functional groups, have been reported to be very sensitive to changes in the environmental $\mathrm{pH}$. The swelling properties of the ionic hydrogels are unique due to the ionization of their pendent functional groups. Hydrogels containing basic functional groups is found to have increased swelling activity in acidic conditions and lesser activity in basic conditions but on the other hand $\mathrm{pH}$ sensitive anionic hydrogels shows low swelling activity in acidic medium and very high activity in basic medium. It appears that with increased cross-linking, diffusion of hydrolyzing agents in the polymer network is reduced and the hydrolysis rate is slower.

\section{Results and discussion}

The chemical attachment of a drug to a synthetic polymer is one means of increasing the duration of activity through slow-release, or target-directing drugs in the body [7]. The chemical structure of the monomeric units along the polymer chain is an important factor in hydrolytic behavior of polymeric prodrugs. One important way for drug delivery systems is the use of dosage forms coated with a $\mathrm{pH}$-sensitive coating $[8,9]$. In this work we created novel macromolecule systems with novel architecture 
that can be used in drug delivery systems. The colonic drugs delivery is not only used for local diseases associated with the colon but also for delivery of proteins and therapeutic peptides. These classes of drugs require the polymers that have architecture which swell minimally under acidic conditions but extensively in basic intestinal medium $[10,11]$. Our polymers have an architecture which show great difference in swelling behavior at $\mathrm{pH}=1$ and 7.4. We used divinylbenzene (DVB), and 1,2-bis(vinylphenyl)ethane (BVPE) as cross-linking agents. These agents were used for modification of polymers such as polystyrene. In this way we incorporated them in terpolymers and prepared new macromolecule systems for other applications. BVPE was prepared from coupling reaction of Grignard reagent with parachloromethylstyrene (Scheme1).<smiles>C=Cc1ccc(CCl)cc1</smiles>

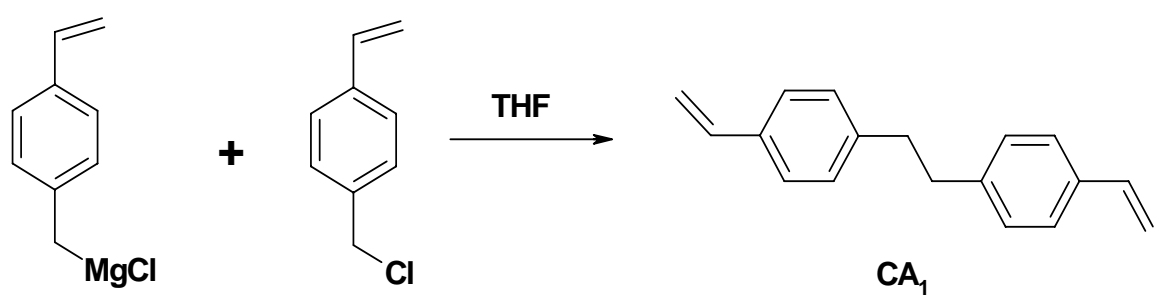

Scheme 1. Preparation of 1,2-bis(vinylphenyl)ethane (BVPE).

These compounds as cross-linking agents were polymerized with TBSiEMA, TESIEMA, and MAA in various ratios to produce copolymer with hydrogel properties; the composition of various reagents used is shown in Table 1.

Tab. 1. The molar composition and $\mathrm{T}_{\mathrm{g}}$ data of copolymers.

$$
\text { Copolymer t-Bu: MAA: \%CA } \mathrm{T}_{\mathrm{g}}^{\circ}(\mathrm{C}) \text { Copolymer } \quad \mathrm{TEt}: \mathrm{MAA}: \% \mathrm{CA} \quad \mathrm{T}_{\mathrm{g}}^{\circ}(\mathrm{C})
$$

\begin{tabular}{|c|c|c|c|c|c|c|c|c|c|}
\hline$P-1$ & 1 & 1 & $5 \%(\mathrm{CA} 1)$ & 159 & $P-7$ & 1 & 1 & $5 \%(\mathrm{CA} 1)$ & 81 \\
\hline P-2 & 1 & 1 & $5 \%(C A 2)$ & 166 & P-8 & 1 & $: 1$ & $5 \%(C A 2)$ & 159 \\
\hline P-3 & 3 & 1 & $5 \%(\mathrm{CA} 1)$ & 124 & P-9 & 3 & 1 & $5 \%(\mathrm{CA} 1)$ & 111 \\
\hline P-4 & 3 & 1 & $5 \%(C A 2)$ & 181 & P-10 & 3 & $: 1$ & $5 \%(C A 2)$ & 158 \\
\hline$P-5$ & 1 & 3 & $5 \%(C A 1)$ & 146 & P-11 & 1 & 3 & $5 \%(C A 1)$ & 159 \\
\hline P-6 & 1 & 3 & $5 \%(\mathrm{CA} 2)$ & 181 & P-12 & 1 & 3 & $5 \%(\mathrm{CA} 2)$ & 125 \\
\hline
\end{tabular}


The synthesis route described above for silyl monomers and cross-linked copolymer containing silyl group is sketched in Scheme 2. Incorporation of silyl group to macromolecules can cause hydrogel properties [12]. By Attaching of silyl methacrylates i.e. TBSiEMA, TESiEMA in polymer chain, drug delivery property is modified and they have good difference in swelling at $\mathrm{pH}=1$ and $\mathrm{pH}=7.4$ [13]. In addition to above properties, it is shown that there is a balance between hydrophilic and lipophilicity of polymer in the loading of drugs to polymers. Since most of drug molecules have a lipophile structure, the presence of lipophile groups on the polymer chain increases drug loading. The silyl groups that we used have lipophile property; therefore synthesis of silyl derivative of polymers and copolymers could be important in drug delivery systems.

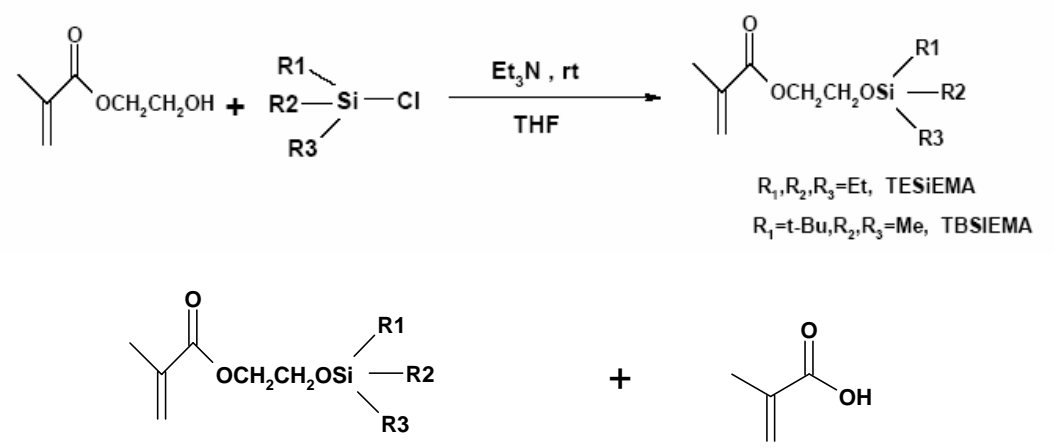

$\mathbf{R}_{1}, \mathbf{R}_{2}, \mathbf{R}_{3}=E t$, TESiEMA

$R_{1}=t-B u, R_{2}, R_{3}=M e$, TBSIEMA
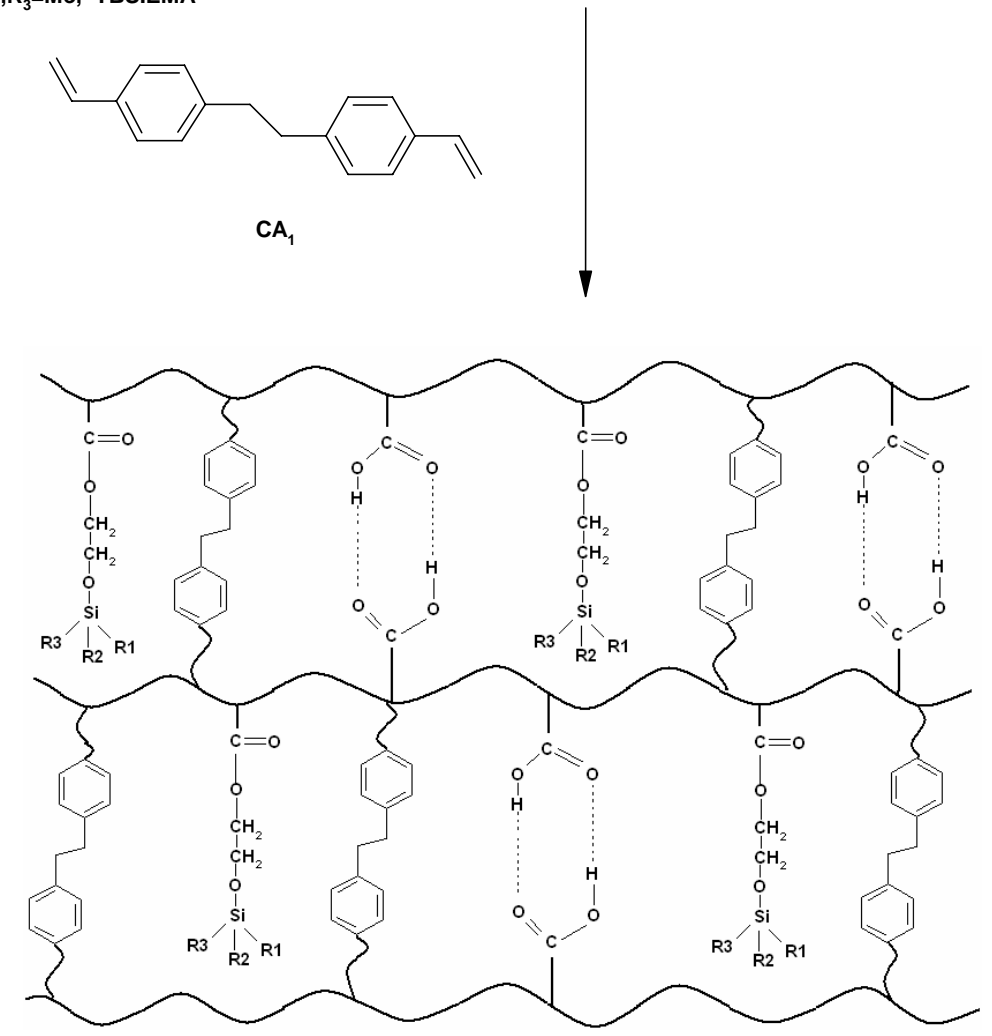

Scheme 2. Preparation of silyl monomers and cross-linked copolymer containing silyl group. 


\section{Thermal behaviour}

The glass transition temperature $\left(T_{g}\right)$ was determined from differential scanning calorimetry (DSC) thermograms. These values are given in Table 1. It appears that with increase in cross-linking degree, the flexibility of chains and the ability of the chains to undergo segmental motion decreases, which in turn increases the $T_{g}$ values [14]. As shown in Table 1, $\mathrm{T}_{\mathrm{g}}$ for polymers using $\mathrm{CA}_{1}$ decreases with reference to $\mathrm{CA}_{2}$. These differences can be attributed to structure of the two crosslinking agents where the flexibility of the $C A_{1}$ when compared to $C A_{2}$ is high enough to undergo segmental motion. Presence of silyl groups in our macromolecule systems not only reduces the hydrogen bonding between the polymer chains in methacrylate monomers but also increases the flexibility of hard polymers by acting as a plasticizer and reduces its $T_{g}$. Also, increasing of MAA in polymer systems causes increase in $T_{g}$ value. This difference is due to hydrogen bonding in MAA. Therefore, in our systems there is enough balance in $T_{g}$ values of polymers.

\section{Drug loading in hydrogels}

$70 \mathrm{mg}$ of hydrogels $\left(\mathrm{P}_{1}, \mathrm{P}_{2}, \mathrm{P}_{3}, \mathrm{P}_{4}, \mathrm{P}_{5}, \ldots, \mathrm{P}_{12}\right)$ was placed in $10 \mathrm{ml}$ of 5-ammino-2hydroxybenzoicacid (5-ASA) to suck up the total amount of the drug solution. After approximately $60 \mathrm{~min}$, the completely swollen hydrogels loaded with 5-ASA were placed in desiccators and dried under vacuum at room temperature.

\section{-Determination of amount of drug entrapped}

The amount of drug entrapped in the hydrogels was determined by an indirect method. After the gel preparation, the washing with methanol was collected and tested using UV-Vis spectroscopy. The difference between the amount of drug initially employed and the drug content in the washing is taken as an indication of the amount of drug entrapped. Hydrogen bonding is a key contributor to the specificity of intramolecular and intermolecular interactions in biological systems and polar drugs. The hydrogen-bonding and electrostatic interaction increased with MAA content in the copolymer networks. 5-ASA molecule has a tendency to attach to polar groups because of hydrogen-bonding. Existence of polar functional groups such as carboxylic acid is not only needed for loading drug on the polymer but also for $\mathrm{pH}$ sensitive properties of polymer.

\section{Characterization of monomers and polymers}

The monomers of TBSiEMA, TESiEMA, and cross-linking of $\mathrm{CA}_{1}$ were identified by ${ }^{1} \mathrm{H}-\mathrm{NMR}$ and IR spectroscopy. These data are given in experimental section for these compounds. Network polymers were characterized by Infrared spectroscopy. The resulting network polymers swell and become soft if they are exposed to solvents such as $\mathrm{H}_{2} \mathrm{O}$ and most other organic solvents without dissolving. In the FT-IR spectra, absorption of C-Si bond appeared in region of $1258-1270$ and $837-900 \mathrm{~cm}^{-1}$ which refers to stretching, and bending vibration respectively, absorption in $1730 \mathrm{~cm}^{-1}$ refers to stretching carbonyl bond in network polymers with silyl methacrylate linkage (TBSiEMA, TESiEMA), in $1710 \mathrm{~cm}^{-1}$ refers to carbonyl group of MAA, and absorption in $2500-3300 \mathrm{~cm}^{-1}$ is referring to $\mathrm{OH}$ bond in MAA. In this way we characterized the monomer units in cross-linked copolymers. 


\section{In vitro release studies}

The copolymers (10 mg) were poured into $3 \mathrm{ml}$ of simulated gastric fluid (SGF) in pH of 1 or simulated intestinal fluid (SIF) in $\mathrm{pH}$ of 7.4. The mixture was introduced into a cellophane membrane dialysis bag. The bags were closed and transferred to a flask containing $20 \mathrm{ml}$ of the same solution maintained at $37^{\circ} \mathrm{C}$. The external solution was continuously stirred, and $3 \mathrm{ml}$ samples were removed at selected intervals. The volume removed was replaced with SGF or SIF. The hydrolyzed sample was analyzed by UV spectrophotometer.

\section{-Drug Release by Hydrolysis of Polymer Bonded Drugs}

Silicones, or silicon polymers, have a number of advantages for use in medical devices and drug delivery systems. Silicone's hydrophobicity makes it particularly suitable for the delivery of lipophilic drugs. Silicones generally are impermeable to polar compounds, which results in low permeability of water-soluble drugs. Drug release profiles from polymers containing silicone are affected by the physiochemical properties of the drug and any additives used in the formulation, as well as the polymer geometry and the drug and additive loading [15]. One way to affect the release of both lipophilic and hydrophilic drugs has been to disperse various compounds within the silicone. The MZ molecules have a tendency to attach to polar groups due to hydrogen-bonding. Existence of polar functionally groups as carboxylic acid is needed not only for loading of drug on the polymer but also for $\mathrm{pH}$-sensitive properties of polymer.

In order to study potential application of polymer bonded drugs (PBDs) containing 5aminosalicylic acid as a pharmaceutically active compound, we have studied the hydrolysis behavior of the polymers under physiological conditions. Although the polymers were not soluble in water, they were dispersed in a buffer solution, and the hydrolysis was evaluated as a heterogeneous system. The degree of hydrolysis of the network polymers containing mesalasine as a function of time are shown in Figure 1.

The concentration of mesalasine released at selected time intervals was determined by UV spectrophotometry at $210 \mathrm{~nm}$. The order of hydrolysis in this series was significantly affected by polymer composition. It appears that the degree of hydrolysis network polymers depends on the degree of reticulation. Increased cross-linking increases the reticulation of the polymer, thus reducing the diffusion of the hydrolyzing agents from the network's polymer which in turn reduces the hydrolysis rate. On the other hand, a higher difference in hydrolysis rate for polymers at $\mathrm{pH} 1$ and 7.4 can be related to the number of carboxylic acid groups on monomeric units along the polymer chain. At pH 7.4 with complete ionization and increased hydrophilicity of the polymer, the diffusion of the hydrolyzing agents on the polymer is increased and the hydrolysis rate also increases. 

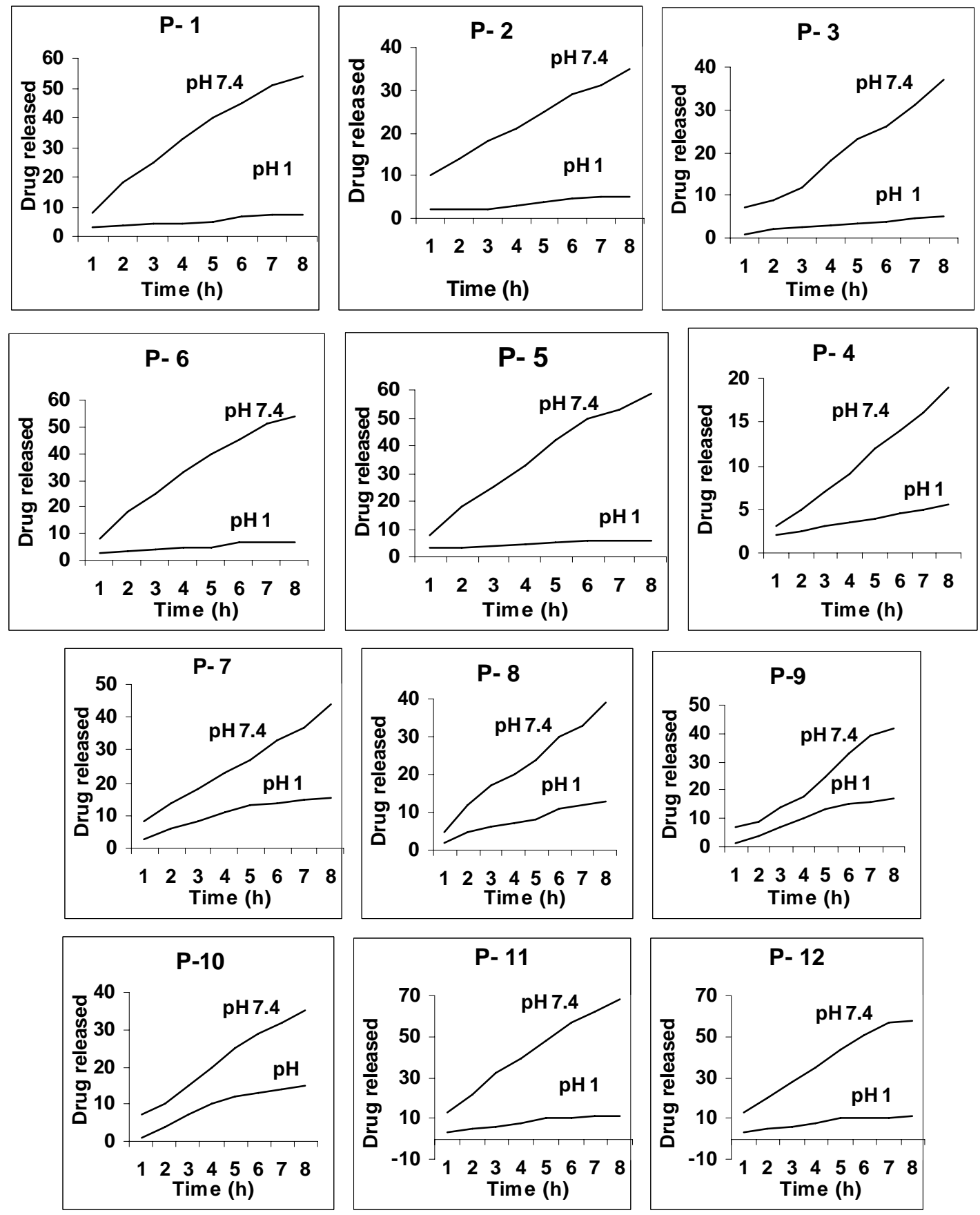

Fig. 1. Release of 5-ASA from polymeric carriers as a function of time at $37^{\circ} \mathrm{C}$.

\section{Conclusions}

The terpolymeric hydrogels containing silyl pendant groups with $\mathrm{pH}$-sensitive swelling properties were synthesized by free radical copolymerization. The oral administration of drugs is the most attractive route, but for reducing gastrointestinal side effects and degradation of drugs, the large intestine may be optimal for drug delivery because of the high residence time and low digestive enzymatic activity. The hydrolytic behaviour of the hydrogels was dependent on the content of MAA groups which caused a decrease in releasing of SGF or an increase in SIF. Incorporation of MAA made the hydrogels $\mathrm{pH}$-dependent and the transition between the swollen and the collapsed states occurred at high and low $\mathrm{pH}$. The swelling ratios of the hydrogels 
increased at $\mathrm{pH} 7.4$, but decreased at $\mathrm{pH} 1$ with increasing incorporation of MAA. When the cross-linking ratio of the copolymer increased, the releasing degree of the hydrogels decreased at both pHs 1 and 7.4. Therefore by placing lipophilic silyl groups in $\mathrm{pH}$-sensitive hydrogel and regulating the cross-linking degree, we can obtain novel $\mathrm{pH}$-sensitive polymer systems with novel physical and chemical properties and new applications. For example, the $\mathrm{pH}$-sensitive polymer systems with silyl side chain substituents can be used as fluid separation membranes and oral drug delivery carriers. Our macromolecule systems contain two new cross-linking agents that customized network polymers for drug delivery.

\section{Experimental:}

Synthesis of monomers and copolymers were carried out under dry argon to exclude oxygen and moisture from the reaction systems.

\section{Materials}

The solvents and reagents were purchased from Merck and Aldrich Co. THF was dried by standard method and divinylbenzene, $\mathrm{Et}_{3} \mathrm{SiCl}, \mathrm{Me}_{2}$ tert $(\mathrm{Bu}) \mathrm{SiCl}$ used as received. Initiator $\alpha, \alpha^{\prime}$-azobis(isobutyronitrile)(AIBN) was purified by crystallization from methanol.

\section{Measurements}

${ }^{1} \mathrm{H}$-NMR spectra were recorded on a Brucker $400 \mathrm{AC}$ spectrometer in $\mathrm{CDCl}_{3}$. The IR spectra were recorded on a Shimadzu FT IR-408 spectrophotometer. The DSC curves were obtained on a TGA/SDTA 851 calorimeter at heating and cooling rates of $10^{\circ} \mathrm{C} / \mathrm{min}$ in air.

\section{Monomers synthesis}

\section{2-Propenoic acid, 2-methyl-2-[(triethylsilyl)oxy] ethyl ester: (TESiEMA)}

A solution of $6.7 \mathrm{~g}(23 \mathrm{mmol})$ triethylchlorosilane in $10 \mathrm{ml}$ dried THF was added drop wise to a mixture of $3 \mathrm{~g}$ (23 mmol) HEMA and $3.5 \mathrm{~g} \mathrm{(34} \mathrm{mmol)} \mathrm{triethylamine} \mathrm{in} 50 \mathrm{ml}$ THF under dry argon at room temperature. After $6 \mathrm{~h}$ stirring at room temperature the reaction mixture was filtered, the THF was removed under reduced pressure to produce a nearly colorless oily residue. The residue was chromatographed over silica gel by $\mathrm{CH}_{2} \mathrm{Cl}_{2}$ to yield TESiEMA (40\%). IR (neat, $\mathrm{cm}^{-1}$ ):2940, 1722, 1640, 1257, 840.

${ }^{1} \mathrm{H}-\mathrm{NMR}\left(\mathrm{CDCl}_{3}, \mathrm{ppm}\right): 0.06\left(\mathrm{~s}, 6 \mathrm{H},-\mathrm{CH}_{2}\right), 0.9\left(\mathrm{~s}, 9 \mathrm{H},-\mathrm{CH}_{3}\right), 1.9\left(\mathrm{~s}, 3 \mathrm{H},-\mathrm{CH}_{3}\right), 3.82(\mathrm{t}$, $\left.2 \mathrm{H},-\mathrm{OCH}_{2}\right), 4.2\left(\mathrm{t}, 2 \mathrm{H},-\mathrm{COOCH}_{2}\right), 5.53\left(\mathrm{~s}, 1 \mathrm{H}, \mathrm{CH}_{2}=\right), 6.1\left(\mathrm{~s}, 1 \mathrm{H}, \mathrm{CH}_{2}=\right)$.

Propenoic acid, 2-methyl-2-[(t-butyldimethyl)silyl)oxy] ethyl ester: (TBSiEMA)

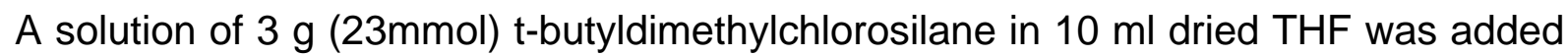
dropwise to a mixture of $3 \mathrm{~g} \mathrm{(23} \mathrm{mmol)HEMA} \mathrm{and} 3.5 \mathrm{~g}(34 \mathrm{mmol})$ triethylamine in 50 $\mathrm{ml}$ THF under dry argon at room temperature. After $24 \mathrm{~h}$ stirring at room temperature the reaction mixture was filtered, the THF was removed under reduced pressure to produce a nearly colorless oily residue. The residue was chromatographed over silica gel by $\mathrm{CH}_{2} \mathrm{Cl}_{2}$ to yield TBSiEMA (35\%).

IR (neat, cm ${ }^{-1}$ ): 2930, 2958, 1722, 1640, 1257, 1168, 1131, 837. 
${ }^{1} \mathrm{H}-\mathrm{NMR}\left(\mathrm{CDCl}_{3}, \mathrm{ppm}\right): 0.06\left(\mathrm{~s}, 6 \mathrm{H},-\mathrm{CH}_{2}\right), 0.87(\mathrm{~s}, 9 \mathrm{H}, \mathrm{t}-\mathrm{Bu}), 1.94\left(\mathrm{~s}, 3 \mathrm{H},-\mathrm{CH}_{3}\right), 3.84$ (t, $\left.2 \mathrm{H}, \mathrm{OCH}_{2}\right), 4.2\left(\mathrm{t}, 2 \mathrm{H},-\mathrm{COOCH}_{2}\right), 5.55\left(\mathrm{~s}, 1 \mathrm{H}, \mathrm{CH}_{2}=\right), 6.1\left(\mathrm{~s}, 1 \mathrm{H}, \mathrm{CH}_{2}=\right)$.

\section{Cross linking agent synthesis}

\section{1,2-Bis(vinylphenyl)ethane (BVPE)}

$6.10 \mathrm{~g} \mathrm{(40} \mathrm{mmol)} \mathrm{4-Chloromethylstyrene} \mathrm{dissolved} \mathrm{in} 30 \mathrm{ml}$ THF was added to $1.07 \mathrm{~g}$ (44 mmol) magnesium turning under argon gas at $37^{\circ} \mathrm{C}$ for $2 \mathrm{~h}$ to produce Grignard reagent. Then $6.01 \mathrm{~g} \mathrm{(40} \mathrm{mmol)} \mathrm{4-chloromethylstyrene} \mathrm{dissolved} \mathrm{in} 15 \mathrm{ml}$ THF was added drop wise to Grignard solution and stirred at the same temperature for $2 \mathrm{~h}$. The precipitate was filtered, the THF was removed under reduced pressure and the residue was chromatographed over silica gel by $n$-Hexane to yield (20\%) residue of 1,2-bis(vinylphenyl)ethane (BVPE).

IR (neat, cm-1): 3083, 2920, 1629, 1509, 1348, 912, 829.

${ }^{1} \mathrm{H}-\mathrm{NMR}\left(\mathrm{CDCl}_{3}, \mathrm{ppm}\right): 2.96\left(\mathrm{~s}, 2 \mathrm{H},-\mathrm{CH}_{2}\right), 5.25-5.28\left(\mathrm{q}, 1 \mathrm{H},=\mathrm{CH}_{2}\right), 5.75-5.80\left(\mathrm{q}, 1 \mathrm{H},=\mathrm{CH}_{2}\right)$, 6.72-6.79(q,1H,CH=),7.18(d,2H,C-H Ar), 7.38(d,2H,C-H Ar). (See Figure 2)

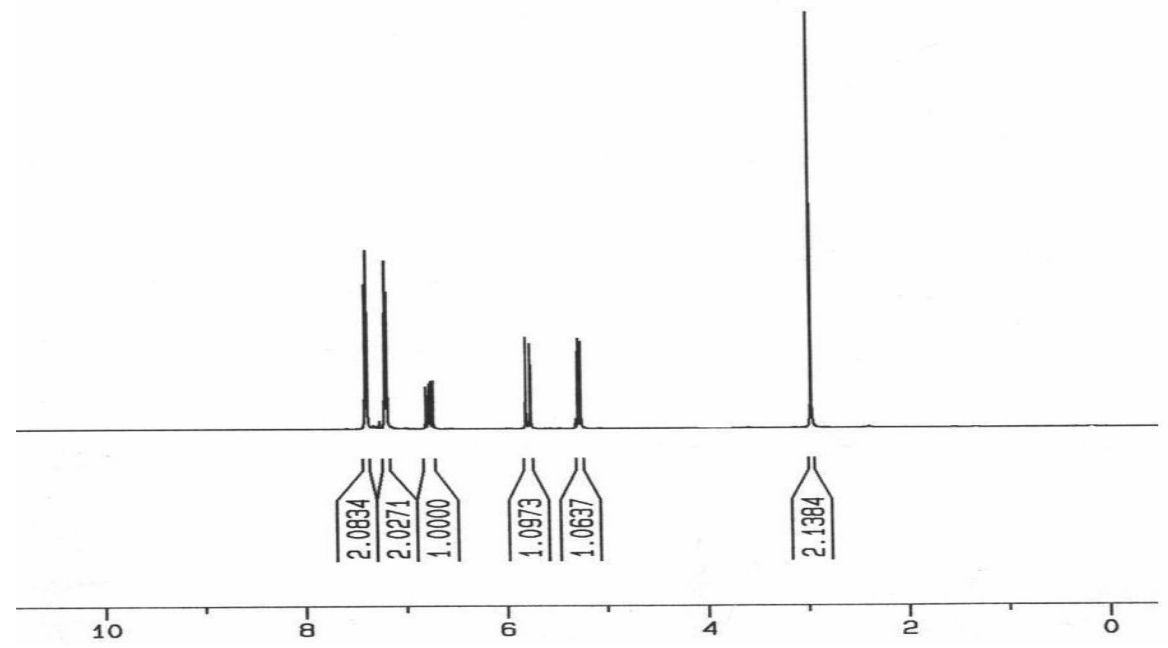

Fig. 2. ${ }^{1} \mathrm{H}$ NMR spectrum of 1,2-bis(vinylphenyl)ethane (BVPE).

\section{General processes for cross-linked copolymerization}

In pyrex glass ampoules, the mixture consisting of silyl monomers and MAA in different molar ratio and specific moles of $\mathrm{CA}_{1}$ (BVPE) or $\mathrm{CA}_{2}$ (DVB) $(5 \%)$ were polymerized at $60-70{ }^{\circ} \mathrm{C}$ in the oil bath using $\alpha, \alpha^{\prime}$-azobis(isobutyronitrile) (AIBN) as initiator $([\mathrm{l}]=0.02)$. The reaction conditions are shown in Table 1. After the specified time $(72 \mathrm{~h})$, the precipitated network polymer was collected, washed with methanol and dried in vacuum. The absorption of C-Si bond appeared in region of 1254 and $835 \mathrm{~cm}^{-1}$ in FT-IR spectrum.

\section{References}

[1] Stevens, M. P. Polymer Chemistry: An Introduction, 3rd ed.; Oxford University Press: New York, NY, 1999; Aldrich Cat. No. Z41, 255-4.

[2] DeBord, T. J. Jr.; Schick, M. Ink World, April 1999, 47. 
[3] Wicks, Z. W. Jr.; Jones, F. N.; Pappas, S. P. Organic Coatings: Science and Technology, 2nd ed.; Wiley-Interscience: New York,NY, 1999; Aldrich Cat. No. Z41, 244-9.

[4] Jing, Z.; Nicholas, A. Macromolecules, 2000, (33), 102-107.

[5] Jones, A. T.; Gumbleton, M.; Duncan, R. Advanced Drug Delivery Reviews 55, 2003, 1353-1357.

[6] Thanou, M.; Duncan, R. Current Opinion in Investigational Drugs 2003, 4(6), 701709.

[7] Akashi, M. In: Biomedical Application of Polymeric Materials. Tsuruta, T.; Hayashi, T.; Ishihara, K.; Kataoka, K.; Kimura, Y. (Eds). 1993, 372-406. CRC Press, Boca Raton, FL.

[8] Dew, M. J.; Hughes, P. J.; Lee, M. G.; Evans, B.K.; Rhodes, J. Journal Clin. Pharmacol. 1982, 14, 405-408.

[9] Schroder, K. W.; Termaine, W. J.; Journal Med. 1987, 317, 1625-1629.

[10] Langer, R. Accounts of Chemical Research, 1993, 26, 537-542

[11] Swarbrick, J.; Boylan, J. (ed), Controlled- and Modulated-Release Drug-Delivery Systems. Encyclopedia of Pharmaceutical Technology. Marcel Dekker, Inc, 1990.

[12] Brahim, S.; Narinesingh, D.; Guiseppi-Elie, A. Biomacromolecules. 2003, 4(5), 1224-31.

[13] Mahkam, M.; Mohammadi, R.; Assadi, M.G.; Ranaei-Siadat, S.O.; Barshan, M.; Siadat, R. Silicon Chemistry, 2005, 1-8.

[14] Mahkam, M.; Sharifi, N.; Entezami, A. A. J. Bioact. Coomp. Polm. 2000, 15, 396405.

[15] Kajihara., M. etal., Chemical and Pharmacetical Bulletin, 51, 11-14. 